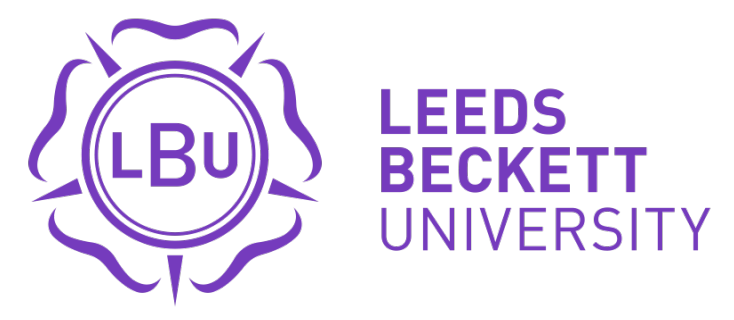

Citation:

Laganà, MM and Shepherd, SJ and Cecconi, P and Beggs, CB (2017) Intracranial volumetric changes govern cerebrospinal fluid flow in the Aqueduct of Sylvius in healthy adults. Biomedical Signal Processing and Control, 36. pp. 84-92. ISSN 1746-8094 DOI: https://doi.org/10.1016/j.bspc.2017.03.019

Link to Leeds Beckett Repository record:

https://eprints.leedsbeckett.ac.uk/id/eprint/3653/

Document Version:

Article (Accepted Version)

Creative Commons: Attribution-Noncommercial-No Derivative Works 4.0

The aim of the Leeds Beckett Repository is to provide open access to our research, as required by funder policies and permitted by publishers and copyright law.

The Leeds Beckett repository holds a wide range of publications, each of which has been checked for copyright and the relevant embargo period has been applied by the Research Services team.

We operate on a standard take-down policy. If you are the author or publisher of an output and you would like it removed from the repository, please contact us and we will investigate on a case-by-case basis.

Each thesis in the repository has been cleared where necessary by the author for third party copyright. If you would like a thesis to be removed from the repository or believe there is an issue with copyright, please contact us on openaccess@leedsbeckett.ac.uk and we will investigate on a case-by-case basis. 


\section{Intracranial volumetric changes govern cerebrospinal fluid flow in the Aqueduct of Sylvius in healthy adults}

Maria Marcella Laganà ${ }^{{ }^{*}}$, Simon J Shepherd ${ }^{2}$, Pietro Cecconi ${ }^{1}$ and Clive B Beggs ${ }^{3,4}$

1. Fondazione Don Carlo Gnocchi ONLUS, IRCCS S. Maria Nascente. Milan, Italy

2. Medical Biophysics Laboratory, University of Bradford, Bradford, BD7 1DP, UK

3. Buffalo Neuroimaging Analysis Center, Department of Neurology, School of Medicine and Biomedical Sciences, University at Buffalo, 100 High St., Buffalo, NY 14203, NY, USA;

4. Institute for Sport, Physical Activity and Leisure, Leeds Beckett University, Leeds, LS1 3HE, UK

Running title: Venous volumetric changes influence aqueductal CSF flow

\section{"Corresponding Author:}

Dr Maria Marcella Laganà

IRCCS, Fondazione Don Carlo Gnocchi ONLUS

Via Capecelatro, 66

20148 Milan, Italy

email: mlagana@dongnocchi.it, Tel: +39 0240308074

Sources of Support: This research did not receive any specific grant from funding agencies in the public, commercial, or not-for-profit sectors.

\section{Conflicts of interest}

The authors confirm that this article content has no conflict of interest. 


\title{
Intracranial volumetric changes govern cerebrospinal fluid flow in the Aqueduct of Sylvius in healthy adults
}

\author{
Abstract \\ Purpose: \\ To characterize the intracranial volumetric changes that influence the cerebrospinal fluid \\ (CSF) pulse in the Aqueduct of Sylvius (AoS).
}

Materials and Methods:

Neck MRI data were acquired from 12 healthy adults ( 8 female and 4 males; mean age $=30.9$ years), using a 1.5 Tesla scanner. The intracranial arterial, venous and CSF volumes changes, together with the aqueductal CSF (aCSF) volume, were estimated from flow rate data acquired at $\mathrm{C} 2 / \mathrm{C} 3$ level and in the AoS. The correlations and temporal relationships among these volumes were computed.

Results: The aCSF volumetric changes were strongly correlated $(r=0.967, p<0.001)$ with the changes in intracranial venous volume, whose peak occurred $7.0 \%$ of cardiac cycle $(p=$ 0.023 ) before peak aCSF volume, but less correlated with the intracranial arterial and CSF volume changes $(r=-0.664$ and 0.676 respectively, $p<0.001)$. The intracranial CSF volume change was correlated with the intracranial venous volume change $(r=0.820, p<0.001)$, whose peak occurred slightly before $(4.2 \%$ of $C C, p=0.059)$.

\section{Conclusion:}

The aCSF pulse is strongly correlated with intracranial venous volume, with expansion of the cortical veins occurring prior to aCSF flow towards the third ventricle. Both caudal-cranial aCSF flow and venous blood retention occur when arterial blood volume is at a minimum.

\section{Keywords}

Aqueduct of Sylvius; Phase Contrast MRI; cerebrospinal fluid; venous flow; arterial flow; intracranial vascular model 


\section{Introduction}

In recent years there has been growing interest in the role that the cerebral venous system plays in regulating the biomechanics of fluids inside the cranium, with several studies demonstrating a link between cerebral venous outflow and the dynamics of the cerebrospinal fluid (CSF) in the Aqueduct of Sylvius (AoS) (aqueductal CSF, aCSF) in healthy individuals $[1,2]$ and in multiple sclerosis (MS) patients $[3,4]$. However, although these studies suggest that a link exists between the venous system and the aCSF pulse, the association between the two has not yet been explained. This is largely because the functional behaviour of the cerebral venous system is poorly understood. While the presence of an intracranial windkessel mechanism linking the arterial and CSF pulses is well recognised [5-8], there is much less agreement regarding the interaction of these fluids with the cerebral venous pulse. However, the fact that venous blood exiting the cranium is pulsatile [9, 10], and that an arteriovenous delay (AVD) exists between the peaks in arterial and venous flow [11], suggests that transient storage of blood occurs in the cerebral veins at some point throughout the cardiac cycle (CC). Nevertheless, much still remains unknown about the fluid volumetric changes that occur within the cranium over the $C C$, and the source of the compliance that causes the AVD.

In healthy young adults, blood flow through the cerebral capillary bed is constant and nonpulsatile [5], despite the considerable increase in arterial blood entering the cranium that occurs during systole. This additional blood is transiently stored during systole in the cranial arteries, including the pial arteries that run through the sub-arachnoid space (SAS), which duly expand, displacing CSF out of the cranium into the spinal column. During diastole, when the arterial flow entering the cranium drops below the mean, the blood stored in the arteries is displaced, by elastic recoil, from these vessels into the cerebral capillary bed [12] ensuring that a constant blood flow is maintained through the parenchymal capillaries [5]. As such, the system acts as a windkessel mechanism that not only regulates blood flow within the cranium, but also drives the CSF pulse across the foramen magnum. In comparison to the cervical CSF pulse, the motion of the CSF pulse in the AoS is less well understood. The aCSF pulse, which is closely linked with the motion of the lateral ventricles [13], has an amplitude that is an order of magnitude less than that of the CFS pulse in the upper neck [9]. Because of its central position and proximity to the lateral ventricles, aCSF is often measured in order to assess structural changes that may be occurring in the brain parenchyma. Numerous studies have found increased pulsatility of the aCSF to be associated with MS [3, $4,14,15]$, normal pressure hydrocephalus [16-19], and the formation of dirty appearing white matter [20], suggesting that abnormalities in this pulse may be linked to pathological changes. However, use of the aCSF pulse as a focal indicator of structural changes has been considerably hampered by a general lack of understanding of the factors that influence its motion.

In the study presented here we applied a signal processing methodology to compute the transient volumetric changes that occur in the intracranial fluids throughout the CC. Although many researchers have accurately quantified the cervical blood and CSF flows to and from the cranium using MRI $[9,21-26]$, characterizing the intracranial interactions between these fluids has been more troublesome. However, because the cranium is a rigid container and the fluids enclosed within it are incompressible [27], in theory it should be possible to characterize the behaviour of the various intracranial fluids solely through interpretation of extracranial fluid flow signals acquired from the cervical vessels. To this end, we developed a novel volumetric model, which interpreted fluid flow signals in the neck, to characterize the temporal changes that occur in the intracranial arterial, venous and CSF volumes over the $\mathrm{CC}$. We then used the model to investigate transient changes in intracranial blood and CSF volumes in healthy young adults, with the aim of gaining new insights into the fluid interactions that occur within the cranium and showing how these relate to the motion of the CSF in the AoS. 


\section{Materials and methods}

\subsection{Subjects}

Twelve healthy young adults were enrolled on the study. Inclusion criteria were age $<45$ years and a normal neurological examination. Exclusion criteria were: history of neurological, cardiovascular or metabolic disorders and abnormalities on brain anatomical MRI.

The study was approved by the local Ethics Committee of Don Gnocchi Foundation (Milan, Italy) and a written informed consent was obtained from all subjects prior to study entry.

\subsection{Magnetic resonance acquisition and processing}

Brain and neck MRI were acquired from all subjects, using a 1.5 Tesla scanner (Siemens Magnetom Avanto, Erlangen, Germany), equipped with a 8-channel head coil and a 4channel neck coil. The acquisition protocol consisted of: 1) brain and neck T1-weighted localizer, for the slice positioning of the subsequent sequences; 2) brain 2D dual-echo turbo spin echo to assess any anatomical abnormalities (TR=2,650ms, $T E=28 / 113 \mathrm{~ms}$, echo train length $=5$, flip angle $=150^{\circ}, 50$ interleaved, $2.5-\mathrm{mm}$-thick axial slices with a matrix size $=256 \times 256$, interpolated to $512 \times 512$, FOV $=250 \times 250 \mathrm{~mm}$ ); 3) 2D TOF MR venography of the neck, with a saturation band positioned caudal to the 128 axial slices (in-plane resolution $=0.5 \times 0.5 \mathrm{~mm}^{2}$, slice thickness $=3 \mathrm{~mm}$, distance factor between subsequent slices $=-$ 20, $\mathrm{FOV}=256 \times 192 \mathrm{~mm}^{2}, \mathrm{TR}=26 \mathrm{~ms}, \mathrm{TE}=7.2 \mathrm{~ms}$, flip angle $=70^{\circ}$ ); 4) three retrospective cardiac gated 2D PC MRI for the quantification of arterial and venous flows through the main cervical vessels, CSF flow at between the second and the third cervical vertebrae $(\mathrm{C} 2 / \mathrm{C} 3$ level) (cCSF) and aCSF. The sequence parameters were respectively (blood/cCSF/aCSF): $\mathrm{TR}=33.75 / 30.2 / 30.2 \mathrm{~ms}$, $\mathrm{TE}=5.11 / 7.6 / 7.6 \mathrm{~ms}$, flip angle $=30^{\circ} / 10^{\circ} / 10^{\circ}$, matrix size $=288 \times 384 / 256 \times 256 / 256 \times 256$, pixel size $=0.67 \times 0.67 / 0.62 \times 0.62 / 0.45 \times 0.45 \mathrm{~mm}^{2}$, slice thickness $=4 / 4.5 / 4.5 \mathrm{~mm}$, maximum encoding velocity $(\mathrm{Venc})=50 / 15 / 15 \mathrm{~cm} / \mathrm{s}$. A finger pulse oximeter was used in order to reconstruct different time points in the $C C$, depending on the heart rate frequency. The slice of the PC sequences were positioned perpendicularly to the flow of interest: for the cervical blood flow the sagittal neck localizer and the sagittal and coronal TOF Maximum Intensity Projection images were used for positioning the PC slice at the $\mathrm{C} 2 / \mathrm{C} 3$ level perpendicularly to the internal jugular veins (IJVs) (Figure $1 \mathrm{~A}-\mathrm{C}$ ); for the cCSF flow the sagittal neck localizer was used for positioning perpendicularly to the spinal canal at C2/C3 level (Figure $1 \mathrm{~A}$ ); for the aCSF flow the sagittal brain localizer was used (Figure 2) for positioning perpendicularly to the AoS.

The flow data were processed with FlowQ software [28] by a single trained examiner. For every PC sequence, two kinds of regions of interest (ROls) were manually drawn on the magnitude and phase images: in the structures of interest; for their flow quantification; and in stationary structures for the estimation of the phase offset. The internal jugular veins (IJVs), vertebral veins (VVs), internal carotid arteries, vertebral arteries were segmented on the PC images with Venc $=60 \mathrm{~cm} / \mathrm{s}$ (Figure $1 \mathrm{~d}$ and $\mathrm{e}$ ). The contours were drawn in the first time point and copied for every time point in the $\mathrm{CC}$, adjusting them if needed. When phase aliasing was detected, pixel values were corrected with the algorithm described in a previous work [28]. The CSF (Figure $1 \mathrm{~g}$ ) and the aCSF (Figure $2 \mathrm{c}$ ) were segmented on the PC images with $\mathrm{VENC}=15 \mathrm{~cm} / \mathrm{s}$. The SAS contours were drawn on the phase image corresponding to the time point with the highest caudal flow velocity, since this shows the highest contrast. The magnitude image (Figure $1 \mathrm{f}$ and $2 \mathrm{~b}$ ) was used to increase the confidence of the contours. For every pixel inside the segmented vessels or the CSF, and for every time point, the phase value, corrected by the offset and by the aliasing, was mapped to velocity. According to Siemens convention, positive CSF flow corresponds to cranial direction, negative CSF flow corresponds caudal direction. The flow rate (in $\mathrm{ml} / \mathrm{s}$ ) was computed for each time point, based on the mean velocity and on the cross sectional area of the corresponding structure. 


\subsection{Volumetric model and data analysis}

Flow data and statistical analysis were undertaken using in-house algorithms written in Matlab (Mathworks, Natick, Mass). Arterial flow rate was computed as the sum of the flow in the internal carotid and vertebral arteries and the venous flow rate as the sum of IJVs and VVs flow rates. The arterial, venous, cCSF and aCSF flow rate signals were resampled to 32 data points over the CC. The corresponding cervical flow volume signals were computed, using the trapezoidal method for the discrete-time integration. All the timings were expressed as a fraction of the CC and as a delay from the arterial systole. For each fluid, the flow rate caudal and cranial peak amplitudes and timings were computed. The pulsatility index $(P I)$ and the resistance index $(R I)[29]$ were calculated as the difference between systolic and diastolic peaks normalized by the mean or the systolic peak flow rate, respectively. For the CSF, we computed a caudal and a cranial $R I$, normalizing by the systolic or diastolic peak, respectively. The cCSF and aCSF stroke volumes (i.e. the average CSF volume displaced throughout the $\mathrm{CC}$ ) were calculated by averaging the absolute positive and negative flow rate integrals [9].

In order to characterize the behavior of the intracranial fluidic system we created a computer model in Matlab, a schematic of which is shown in Figure 3. This model comprised three intracranial vascular compartments: an expandable arterial compartment representing the pial arteries, whose volume changed throughout the $\mathrm{CC}$; a central constant-volume compartment, representing the non-pulsatile cerebral vascular bed (i.e. capillaries, arterioles and venules); and an expandable venous compartment representing the cerebral veins, whose volume also changed. In keeping with other researchers [9, 10], we scaled the venous flow rate signal so that its mean matched that of the arterial signal. It was also assumed that the CSF could only interact with the expandable arteries and veins and that the non-pulsatile cerebral vascular bed was completely isolated from the CSF. Incremental changes in the intracranial arterial, venous and CSF volumes were calculated by integrating the PC MRI flow rate measures, assuming that all the changes in fluid flow rate quantified at the cervical level contributed to the intracranial volume changes. This was done by integrating the respective fluid flow rate signals over the $\mathrm{CC}$, as described in equation 1, to compute the volumetric changes $(v c)$ for each of the 32 cardiac fraction divisions.

$$
v c(i)=\int_{i}^{i+1} Q \cdot d t
$$

where: $i$ is a count from 1 to $32 ; \mathrm{Q}$ is the respective fluid flow rate signal, and $d t=C C$ duration divided by 32 time points.

In the model, it was assumed that the intracranial fluid volumetric changes reflected the cumulative changes in the fluid volumes at the cervical level. For the model computations, each cervical fluid volume signal was zero centered (i.e. its mean value was subtracted), as described in equation 2.

$$
v c_{0}(i)=v c(i)-\overline{v c}
$$

where: $v c_{0}$ is the zero centered volumetric change, and is the mean volumetric change.

Finally, the zero-centered fluid volume signal was cumulated, to yield the fluid volumetric changes occurring in the cranium throughout the CC (hereafter called cumulative volume change), as described in equation 3.

$$
v c_{c u m}(i+1)=v c_{0}(i)+v c_{0}(i+1)
$$


where: $v c_{c u m}$ is the cumulated volume change.

We assumed that the changes in intracranial arterial and venous volumes occurred only within the expandable arterial and venous compartments in the model, and that the volume of the central non-pulsatile vascular compartment remaining unchanged.

The timings of the intracranial minimum and maximum cumulative volume change were computed as a fraction of the $\mathrm{CC}$ and as a delay from the minimum arterial value. The arterial minimum corresponded to the beginning of systole, with the maximum in the other cumulative volume change signals corresponding to the point at which the volume of other intracranial fluids started to decrease.

While it was not possible to calculate the absolute intracranial fluid volumes using the model, by utilising the cumulative approach described above it was possible to determine the changes in the intracranial arterial, venous, CSF and aCSF volumetric signals and thus compute the relative changes in respective intracranial fluid volumes over the CC. For each subject, in order to compare the relative changes in the respective volumetric signals, the minimum values of each signal was set to zero, thus ensuring that all the changes were positive.

\subsection{Statistical analysis}

The mean value and standard deviation (SD) across subjects were computed for: the average (across the CC) arterial, venous and CCSF flow rates at the cervical level, and the aCSF flow rate; their caudal and cranial peak timings; the cumulative volume change maximum and minimum timings; the $P I$ and the $R I$. The Wilcoxon test was used to assess if there was a significant delay between the arterial systolic peak and the CCSF and venous peaks; and between the minimum arterial cumulative volume change and the peaks of the remaining intracranial cumulated volumes. Each cumulative volume change curve was averaged across subjects and the correlation between the pair of averaged curves was obtained with Pearson linear correlation. Correlations of $0.30-0.49$ were considered weak, 0.50-0.69 moderated, of 0.70-0.89 strong and higher than 0.90 very strong.

\section{Results}

\subsection{Demographic results}

Demographic analysis of the 12 subjects ( 8 female and 4 males) in the study revealed that the average age of the subjects was 30.9 years old (SD $=7.2$ years; range $20-45$ years).

\subsection{Magnetic resonance imaging results}

The average and SD (across subjects) of the arterial, venous, cCSF, aCSF flow rate curves are shown in Figure 4 and their mean value and SD across time are reported in Table 1. The cervical venous flow rate was slightly less pulsatile (range -9.615 to $-15.619 \mathrm{~mL} / \mathrm{s}$; mean (SD) $\mathrm{Pl}=0.486(0.209)$; mean $(\mathrm{SD}) \mathrm{RI}=0.381(0.127)$ ) than the arterial flow rate (range 8.546 to $20.054 \mathrm{~mL} / \mathrm{s}$; $P I$ mean $(S D)=0.897$ (0.217); mean (SD) $\mathrm{RI}=0.564$ (0.074)), with the cCSF flow fluctuating (range -2.825 to $1.826 \mathrm{~mL} / \mathrm{s}$; mean (SD) caudal $R I=-1.663(0.120)$; cranial $\mathrm{Rl}=2.554(0.275)$ ) around a mean flow rate close to zero. A significant time delay was observed between various cervical flow rate signals. The caudal cCSF peak occurred shortly after the arterial systolic peak (mean $(S D)=5.5 \%(6.3 \%)$ of CC; $p=0.012$ ) and before the systolic venous peak, which occurred $10.9 \%(8.1 \%)$ of CC after the arterial peak; $p<0.001$. The amplitude of the aCSF pulse (range: -0.101 to $0.097 \mathrm{~mL} / \mathrm{s}$; mean (SD) caudal $R I=-$ $1.994(0.234)$; cranial $R I=2.068(0.292)$ ) was an order of magnitude smaller than the cCSF pulse, with the mean stroke volume of the two being $24.2(\mathrm{SD}=15.2) \mu \mathrm{L} /$ beat and $567.3(\mathrm{SD}$ $=222.5) \mu \mathrm{L} /$ beat, respectively. 


\subsection{Intracranial volumetric analysis results}

The intracranial cumulative volume changes for the arterial, venous, and CSF flows, together with the cumulative aCSF volume change are presented in Figure 5. Their correlations are reported in Table 2 and the timings of their minimum and maximum values in Table 3 . The mean peak-to-trough changes in the intracranial arterial and venous volumes were 1259.5 $(\mathrm{SD}=407.6) \mu \mathrm{L}$ and $626.6(\mathrm{SD}=277.6) \mu \mathrm{L}$, respectively, while those for the intracranial CSF and aCSF volumes were $556.8(\mathrm{SD}=216.1) \mu \mathrm{L}$ and $24.0(\mathrm{SD}=15.5) \mu \mathrm{L}$. There was a very strong inverse relationship $(r=-0.997, p<0.001)$ between the intracranial arterial and CSF cumulative volume change signals, with the minimum and maximum intracranial arterial volumes coinciding almost exactly with the peak and trough in the intracranial CSF volume (quantified in Table 3). Likewise, there was a strong inverse relationship $(r=-0.818, p<0.001)$ between the mean intracranial arterial and venous cumulative volume change signals, although in this case, there was a short, but significant, delay $(5.8 \%$ of $C C ; p=0.007)$ between the minimum arterial and the maximum venous volume. By comparison, the mean CSF and venous volumetric signals were positively correlated $(r=0.820, p<0.001)$ with the peak in intracranial venous volume lagging behind the CSF peak by $4.2 \%$ of $C C(p=0.059)$. The aCSF cumulative volume change was very strongly positively correlated with the mean intracranial venous signal $(r=0.967, p<0.001)$, with the peak of the latter being $7.0 \%$ of $C C$ $(p=0.023)$ before the peak of the former, as illustrated in Figure 6 . By comparison the correlations of aCSF cumulative volume change with the mean intracranial arterial cumulative volume change $(r=-0.664, p<0.001)$ and the mean intracranial CSF cumulative volume change $(r=0.676, p<0.001)$ were moderate.

\section{Discussion}

Over the years many studies have investigated the interactions between the various fluids in the intracranial space. Some have investigated the respective fluid flows in the context of neurological pathologies [8, 21, 30,31], while others have sought to understand intracranial fluid dynamics in healthy individuals [1, 9, 32]. Approaches used have varied greatly, from the acquisition of flow and pressure data, with little or no signal processing, through to the construction of complicated lumped parameter electrical analogue models designed to simulate the dynamics of the intracranial space. The former approach is typified by ElSankari and co-workers [9, 21, 24, 25, 32], who in a series of MRI studies used blood and CSF flow data, acquired from strategic locations within the cranium and neck, to compute key metrics such as stroke and pulse volume, and AVD. Bateman and co-workers also used a similar approach to characterise changes in arterial and venous pulse volume and AVD in patients with normal pressure hydrocephalus [8, 11], intracranial hypertension [30], leukoaraiosis [8], vascular dementia [5] and MS [31]. Others have adopted a numerical approach. For example, Martin et al [33] used momentum and continuity equations to produce a hydrodynamic model that coupled the cardiovascular and CSF systems in order to simulate the CSF dynamics in the spinal column. Toro et al [34] also used a hydrodynamic approach to simulate the effect of IJV function on intracranial venous haemodynamics. Linninger et al $[35,36]$ used computational fluid dynamics to simulate intracranial CSF dynamics, while Buishas et al [37] used a complex system of coupled differential equations to simulate CSF production and re-absorption. Other researchers have turned to signal processing in order to model the dynamics of the intracranial space. For example, Wagshul et al [38] and Zou et al [7] took a spectral analysis approach, using Fourier transformation and time-varying transfer functions to model the relationship between the waveforms of intracranial pressure (ICP) and arterial blood pressure. There has also been a long history of the use of lumped parameter and electrical analogue models to simulate intracranial fluid dynamics. In the 1990s, Czosnyka et al [39] and Ursino and Lodi [40] developed electrical analogue models which modelled the fluid interactions in the cranium to simulate fluctuations in ICP. Later, Egnor et al [41] developed a harmonic oscillation model based on an analogue 
AC electrical circuit, which predicted the phase lag of the ICP pulse with respect to the vascular pulse. More recently, other researchers have developed these ideas to produce complex lumped parameter models to simulate: jugular venous waveforms [42]; the hydrodynamics of the cerebrospinal system [10]; and the CSF flow dynamics associated with syringomyelia [43]. Finally, Marcotti et al. [44] used an anatomy-based lumped parameter model to simulate the effect of IJV constriction on intracranial haemodynamics and ICP.

In comparison to the approaches outlined above, relatively few researchers have quantified the volumetric changes that take place in the cranium over the CC. Kim and Kim [45] computed the temporal changes in cerebral arterial and venous volumes in cats in response to visual stimulation. Alperin et al $[22,46]$ developed an algorithm to compute intracranial volume change, which they coupled with Marmarou's function [47] to calculate changes in ICP $[22,48]$. The technique was also used to compute the intracranial volume change in healthy individuals in both the supine and upright positions [49]. A similar approach was used by Baledent et al [50], while Wahlin et al $[51,52]$ built on this work to compute the intracranial arterial volume change. However, while this body of work shares similarities with the methodology used in our study, unlike us, these researchers did not compute the cumulative volumetric changes for the arterial, venous and CSF components individually. By integrating the individual flow rate signals and then zero centring and cumulating the results (i.e. computing the change in volume from the mean), we were able compute the changes in the arterial, venous and CSF volumes in the cranium over the CC, as well as changes in the CSF volume passing through the AoS (Figure 5). This meant that we could then explore the temporal relationships between these various fluids as they accumulated volumetrically in the intracranial space, something that had not previously been done. Through the use of a novel model and relatively simple signal processing techniques, our methodology represents an accessible way to compute volumetric changes in the intracranial fluids, without the need for complex equations. It is easily applicable, requiring just two PC MRI sequences at the cervical level and one reference TOF sequence.

The aggregated flow rate signals shown in Figure 4 are difficult to interpret. However by transforming these, we were able to produce the 'change in volume' signals shown in Figure 5 , which are easier to interpret and yield new insights into the fluid dynamics of the intracranial space. From these it can be seen that the cranium contains a closely coupled fluidic system that is driven by volumetric changes arising from the cardiac pulse. Given that the brain parenchymal tissue is generally thought to be incompressible [53], due to its high water content [27], any expansion in one intracranial fluid will result in the displacement of another, something that is clearly evident from the strong inverse relationship that exists between the arterial and CSF fluids in the cranium (Figure 5). As arterial blood accumulates in the cranium during systole, so it displaces CSF into the spinal column, with the result that the intracranial CSF volume reduces to a minimum. Conversely during diastole, as the arterial blood flow entering the cranium decreases, so the returning CSF replaces the stored arterial blood, with the result that the intracranial CSF volume reaches a maximum at approximately the same time as the intracranial arterial blood volume is at its minimum. Likewise there is a similar inverse relationship between the intracranial venous and arterial volumes, although here the peak venous volume lags behind the minimum arterial volume, suggesting the presence of compliance in the system. As such, this indicates that the response of both the CSF and the venous blood is primarily driven by changes in the volume of the cerebral arteries. Being compliant vessels, these arteries act as an elastic reservoir (i.e. a windkessel mechanism), which expands in systole and then releases stored blood in diastole in order to maintain constant capillary flow [6].

Although the mechanism by which venous blood is transiently stored in the cranium is poorly understood, it is thought that the venous blood is retained in the cortical veins [54, 55], which being thin walled floppy vessels readily expand to accommodate the additional fluid volume [56]. From Table 2 and Figure 5 it can be seen that although there is a strong positive correlation between the venous and CSF volumetric signals, the venous signal lags behind the CSF signal. While both these signals are ultimately driven by changes in the intracranial 
arterial blood volume, the fact that the former lags behind the latter, suggests that the 'coupling' between the arterial and venous compartments is less direct than that between the arterial and CSF compartments. Indeed, it is thought that CSF plays an important intermediary role in coupling the arterial and venous pulses [6-8]. From Figure 5 it can be seen that the mean peak-to-trough change in the intracranial CSF volume represents only about $45 \%$ of the mean peak-to-trough change in the intracranial arterial blood volume. This indicates that the volume of CSF that is displaced from the cranium represents only a fraction of the overall expansion that occurs in the arterial blood vessels during systole. It suggests that as the arterial vessels expand, a further portion of the CSF is displaced within the cranium, which in turn interacts with cerebral veins. Regional displacement of CSF within the sub-arachnoid space (SAS) has been shown to occur throughout the CC [57]. With respect to this, it is thought that the CSF pulse wave, generated by the expanding arterial vessels, compresses the cortical bridging veins that traverse the SAS, causing them to discharge stored venous blood into superior sagittal sinus [6-8]. Greitz [6] estimated that the time taken for the CSF pulse wave to pass from the arteries to the bridging veins is about $30 \mathrm{~ms}$, similar in magnitude to the delay observed by us between the CSF and venous volumetric signals. This delay is indicative of the presence of compliant material in the SAS, something that increases its impedance and slows down the pulse wave [58].

The motion of the CSF flow in the AoS has long been something of a mystery. Greitz [6] suggested that the pulsatility in the AoS might be due to expansion of the cerebral capillaries being transmitted through the parenchyma to the lateral ventricles. However, in recent years a number of studies have demonstrated a link between the motion of the CSF in the AoS and cerebral venous outflow in both healthy individuals [1,2] and MS patients [3, 4], with constriction of the IJVs associated with an increase in amplitude of the aqueductal pulse [1, 2]. The results of the present study reinforce the opinion that there is an association between the aCSF pulse and the intracranial venous volume. From Figure 6, which shows the intracranial venous and aCSF volumetric signals scaled to the same amplitude, it can be seen that the two signals are almost identical in shape $(r=0.967, p<0.001)$, with the aCSF signal occurring shortly after the venous signal. While this indicates a strong correlation between venous blood retention and the motion of the aCSF, it is important to note that this does not necessarily imply causality since both the expansion of the veins and CSF returning into the ventricles are a response to arterial contraction and occur at the nadir of the intracranial pulse pressure wave. Therefore, both phenomena may be a response to a common stimulus. This however does not preclude the possibility that the characteristics of the aCSF pulse might be modified by the functional behaviour of the venous system. Constriction of the cerebral venous outflow is known to increase the volume of blood in the cortical veins [56]. It has also been shown to increase in the amplitude of the aCSF pulse [1, 2], suggesting that an over-accumulation of blood in the cortical veins in some way alters the dynamics of the aqueductal CSF pulse. While the mechanics associated with this are not fully understood, constriction of the IJVs will tend increase the venous blood pressure in the superior sagittal sinus, resulting in reduced CSF absorption [44, 59], something that might affect the aCSF pulse. In addition, as the cortical veins expand in diastole, the overall volume of the SAS will decrease, with the result that the compliance and hydraulic resistance of this space will change. It may therefore be that the expanding venous vessels alter the impedance of the SAS in comparison to that of the AoS, in such a way that CSF is encouraged to change path and flow up the AoS, something that might explain why the aCSF signal lags behind the venous volumetric signal in Figure 6. If an over-accumulation of blood in the cortical veins occurs, say due to constricted outflow, the volume of the SAS would become further reduced, with the result that the amplitude of the aCSF pulse might also increase. Furthermore, because the functional compliance of the cortical bridging veins relies on their ability to empty during systole [11, 60,61], any constriction that inhibits the discharge of venous blood from the cranium has the potential to reduce intracranial compliance - something that might result in a general stiffening of the brain parenchyma as Hatt et al [2] observed when they compressed the IJVs in healthy subjects. 


\section{Conclusions}

To the best of our knowledge, the study presented in this paper is the first to show simultaneously the changes that occur in the arterial, venous and CSF volumes within the cranium in healthy young subjects. We have been able to show that the intracranial venous and CSF volumes respond inversely to changes in the intracranial arterial volume, with the CSF volumetric signal leading the venous signal. We have also been able to show that the motion of the CSF pulse in the AoS is strongly correlated with intracranial venous volume, with the venous volumetric signal leading the aCSF signal. Although this does not demonstrate causality (both venous blood retention and the motion of the CSF in the AoS appear to be primarily a response to changes in the intracranial arterial blood volume), the possibility that the intracranial venous volume influences the aqueductal CSF pulse cannot be excluded. Given that other studies [1,2] have demonstrated an association between increased in amplitude of the aqueductal CSF pulse and constriction of the IJVs, it is recommended that further work be undertaken to investigate the biomechanical interaction between cortical bridging veins and the CSF. 


\section{References}

[1] C.B. Beggs, C. Magnano, S.J. Shepherd, K. Marr, V. Valnarov, D. Hojnacki, N. Bergsland, P. Belov, S. Grisafi, M.G. Dwyer, E. Carl, B. Weinstock-Guttman, R. Zivadinov, Aqueductal cerebrospinal fluid pulsatility in healthy individuals is affected by impaired cerebral venous outflow, J Magn Reson Imaging, 40 (2014) 1215-1222.

[2] A. Hatt, S. Cheng, K. Tan, R. Sinkus, L.E. Bilston, MR Elastography Can Be Used to Measure Brain Stiffness Changes as a Result of Altered Cranial Venous Drainage During Jugular Compression, AJNR Am J Neuroradiol, 36 (2015) 1971-1977.

[3] P. Zamboni, E. Menegatti, B. Weinstock-Guttman, C. Schirda, J.L. Cox, A.M. Malagoni, D. Hojanacki, C. Kennedy, E. Carl, M.G. Dwyer, N. Bergsland, R. Galeotti, S. Hussein, I.

Bartolomei, F. Salvi, R. Zivadinov, The severity of chronic cerebrospinal venous insufficiency in patients with multiple sclerosis is related to altered cerebrospinal fluid dynamics, Funct Neurol, 24 (2009) 133-138.

[4] R. Zivadinov, C. Magnano, R. Galeotti, C. Schirda, E. Menegatti, B. Weinstock-Guttman, K. Marr, I. Bartolomei, J. Hagemeier, A.M. Malagoni, D. Hojnacki, C. Kennedy, E. Carl, C. Beggs, F. Salvi, P. Zamboni, Changes of cine cerebrospinal fluid dynamics in patients with multiple sclerosis treated with percutaneous transluminal angioplasty: a case-control study, J Vasc Interv Radiol, 24 (2013) 829-838.

[5] G.A. Bateman, C.R. Levi, P. Schofield, Y. Wang, E.C. Lovett, The venous manifestations of pulse wave encephalopathy: windkessel dysfunction in normal aging and senile dementia, Neuroradiology, 50 (2008) 491-497.

[6] D. Greitz, Radiological assessment of hydrocephalus: new theories and implications for therapy, Neurosurg Rev, 27 (2004) 145-165; discussion 166-147.

[7] R. Zou, E.H. Park, E.M. Kelly, M. Egnor, M.E. Wagshul, J.R. Madsen, Intracranial pressure waves: characterization of a pulsation absorber with notch filter properties using systems analysis: laboratory investigation, J Neurosurg Pediatr, 2 (2008) 83-94.

[8] G.A. Bateman, Pulse-wave encephalopathy: a comparative study of the hydrodynamics of leukoaraiosis and normal-pressure hydrocephalus, Neuroradiology, 44 (2002) 740-748.

[9] S. Stoquart-EISankari, O. Baledent, C. Gondry-Jouet, M. Makki, O. Godefroy, M.E. Meyer, Aging effects on cerebral blood and cerebrospinal fluid flows, J Cereb Blood Flow Metab, 27 (2007) 1563-1572.

[10] K. Ambarki, O. Baledent, G. Kongolo, R. Bouzerar, S. Fall, M.E. Meyer, A new lumpedparameter model of cerebrospinal hydrodynamics during the cardiac cycle in healthy volunteers, IEEE Trans Biomed Eng, 54 (2007) 483-491.

[11] G.A. Bateman, Vascular compliance in normal pressure hydrocephalus, AJNR Am J Neuroradiol, 21 (2000) 1574-1585.

[12] G.S. Chan, P.N. Ainslie, C.K. Willie, C.E. Taylor, G. Atkinson, H. Jones, N.H. Lovell, Y.C. Tzeng, Contribution of arterial Windkessel in low-frequency cerebral hemodynamics during transient changes in blood pressure, J Appl Physiol (1985), 110 (2011) 917-925. [13] D.C. Zhu, M. Xenos, A.A. Linninger, R.D. Penn, Dynamics of lateral ventricle and cerebrospinal fluid in normal and hydrocephalic brains, J Magn Reson Imaging, 24 (2006) 756-770.

[14] C. Magnano, C. Schirda, B. Weinstock-Guttman, D.S. Wack, E. Lindzen, D. Hojnacki, N. Bergsland, C. Kennedy, P. Belov, M.G. Dwyer, G.U. Poloni, C.B. Beggs, R. Zivadinov, Cine cerebrospinal fluid imaging in multiple sclerosis, J Magn Reson Imaging, 36 (2012) 825-834. [15] Y. Gorucu, S. Albayram, B. Balci, Z.I. Hasiloglu, K. Yenigul, F. Yargic, Z. Keser, F. Kantarci, A. Kiris, Cerebrospinal fluid flow dynamics in patients with multiple sclerosis: a phase contrast magnetic resonance study, Funct Neurol, 26 (2011) 215-222.

[16] P.H. Luetmer, J. Huston, J.A. Friedman, G.R. Dixon, R.C. Petersen, C.R. Jack, R.L. McClelland, M.J. Ebersold, Measurement of cerebrospinal fluid flow at the cerebral aqueduct by use of phase-contrast magnetic resonance imaging: technique validation and utility in diagnosing idiopathic normal pressure hydrocephalus, Neurosurgery, 50 (2002) 534-543; discussion 543-534.

[17] P. Gideon, F. Stahlberg, C. Thomsen, F. Gjerris, P.S. Sorensen, O. Henriksen, Cerebrospinal fluid flow and production in patients with normal pressure hydrocephalus studied by MRI, Neuroradiology, 36 (1994) 210-215. 
[18] D.S. Kim, J.U. Choi, R. Huh, P.H. Yun, D.I. Kim, Quantitative assessment of cerebrospinal fluid hydrodynamics using a phase-contrast cine MR image in hydrocephalus, Childs Nerv Syst, 15 (1999) 461-467.

[19] W.G. Bradley, Jr., D. Scalzo, J. Queralt, W.N. Nitz, D.J. Atkinson, P. Wong, Normalpressure hydrocephalus: evaluation with cerebrospinal fluid flow measurements at MR imaging, Radiology, 198 (1996) 523-529.

[20] C.B. Beggs, C. Magnano, S.J. Shepherd, P. Belov, D.P. Ramasamy, J. Hagemeier, R. Zivadinov, Dirty-Appearing White Matter in the Brain is Associated with Altered

Cerebrospinal Fluid Pulsatility and Hypertension in Individuals without Neurologic Disease, $\mathrm{J}$ Neuroimaging, 26 (2016) 136-143.

[21] S. El Sankari, C. Gondry-Jouet, A. Fichten, O. Godefroy, J.M. Serot, H. Deramond, M.E. Meyer, O. Baledent, Cerebrospinal fluid and blood flow in mild cognitive impairment and Alzheimer's disease: a differential diagnosis from idiopathic normal pressure hydrocephalus, Fluids Barriers CNS, 8 (2011) 12.

[22] N.J. Alperin, S.H. Lee, F. Loth, P.B. Raksin, T. Lichtor, MR-Intracranial pressure (ICP): a method to measure intracranial elastance and pressure noninvasively by means of MR imaging: baboon and human study, Radiology, 217 (2000) 877-885.

[23] P.B. Raksin, N. Alperin, A. Sivaramakrishnan, S. Surapaneni, T. Lichtor, Noninvasive intracranial compliance and pressure based on dynamic magnetic resonance imaging of blood flow and cerebrospinal fluid flow: review of principles, implementation, and other noninvasive approaches, Neurosurg Focus, 14 (2003) e4.

[24] S. El Sankari, M. Czosnyka, P. Lehmann, M.E. Meyer, H. Deramond, O. Baledent, Cerebral Blood and CSF Flow Patterns in Patients Diagnosed for Cerebral Venous Thrombosis - An Observational Study, J Clin Imaging Sci, 2 (2012) 41.

[25] S. Stoquart-El Sankari, P. Lehmann, C. Gondry-Jouet, A. Fichten, O. Godefroy, M.E. Meyer, O. Baledent, Phase-contrast MR imaging support for the diagnosis of aqueductal stenosis, AJNR Am J Neuroradiol, 30 (2009) 209-214.

[26] M.M. Lagana, A. Chaudhary, D. Balagurunathan, D. Utriainen, P. Kokeny, W. Feng, P. Cecconi, D. Hubbard, E.M. Haacke, Cerebrospinal fluid flow dynamics in multiple sclerosis patients through phase contrast magnetic resonance imaging, Curr Neurovasc Res, 11 (2014) 349-358.

[27] L.E. Bilston, Brain tissue mechanical properties, in: K. Miller (Ed.) Biomechanics of the brain, Springer, New York, 2011, pp. 69-89.

[28] E.M. Haacke, W. Feng, D. Utriainen, G. Trifan, Z. Wu, Z. Latif, Y. Katkuri, J. Hewett, D. Hubbard, Patients with multiple sclerosis with structural venous abnormalities on MR imaging exhibit an abnormal flow distribution of the internal jugular veins, J Vasc Interv Radiol, 23

(2012) 60-68 e61-63.

[29] R.O. Bude, J.M. Rubin, Relationship between the resistive index and vascular compliance and resistance, Radiology, 211 (1999) 411-417.

[30] G.A. Bateman, Vascular hydraulics associated with idiopathic and secondary intracranial hypertension, AJNR Am J Neuroradiol, 23 (2002) 1180-1186.

[31] G.A. Bateman, J. Lechner-Scott, R.A. Lea, A comparison between the pathophysiology of multiple sclerosis and normal pressure hydrocephalus: is pulse wave encephalopathy a component of MS?, Fluids Barriers CNS, 13 (2016) 18.

[32] S. Stoquart-Elsankari, P. Lehmann, A. Villette, M. Czosnyka, M.E. Meyer, H. Deramond, O. Baledent, A phase-contrast MRI study of physiologic cerebral venous flow, J Cereb Blood Flow Metab, 29 (2009) 1208-1215.

[33] B.A. Martin, P. Reymond, J. Novy, O. Baledent, N. Stergiopulos, A coupled hydrodynamic model of the cardiovascular and cerebrospinal fluid system, Am J Physiol Heart Circ Physiol, 302 (2012) H1492-1509.

[34] E.F. Toro, L.O. Muller, M. Cristini, E. Menegatti, P. Zamboni, Impact of Jugular Vein Valve Function on Cerebral Venous Haemodynamics, Curr Neurovasc Res, 12 (2015) 384397.

[35] A.A. Linninger, C. Tsakiris, D.C. Zhu, M. Xenos, P. Roycewicz, Z. Danziger, R. Penn, Pulsatile cerebrospinal fluid dynamics in the human brain, IEEE Trans Biomed Eng, 52 (2005) 557-565. 
[36] A.A. Linninger, M. Xenos, D.C. Zhu, M.R. Somayaji, S. Kondapalli, R.D. Penn, Cerebrospinal fluid flow in the normal and hydrocephalic human brain, IEEE Trans Biomed Eng, 54 (2007) 291-302.

[37] J. Buishas, I.G. Gould, A.A. Linninger, A computational model of cerebrospinal fluid production and reabsorption driven by Starling forces, Croat Med J, 55 (2014) 481-497.

[38] M.E. Wagshul, E.J. Kelly, H.J. Yu, B. Garlick, T. Zimmerman, M.R. Egnor, Resonant and notch behavior in intracranial pressure dynamics, J Neurosurg Pediatr, 3 (2009) 354-364.

[39] M. Czosnyka, S. Piechnik, H.K. Richards, P. Kirkpatrick, P. Smielewski, J.D. Pickard, Contribution of mathematical modelling to the interpretation of bedside tests of cerebrovascular autoregulation, J Neurol Neurosurg Psychiatry, 63 (1997) 721-731.

[40] M. Ursino, C.A. Lodi, A simple mathematical model of the interaction between intracranial pressure and cerebral hemodynamics, J Appl Physiol (1985), 82 (1997) 12561269.

[41] M. Egnor, A. Rosiello, L. Zheng, A model of intracranial pulsations, Pediatr Neurosurg, 35 (2001) 284-298.

[42] J. Kim, N.A. Thacker, P.A. Bromiley, A. Jackson, Prediction of the jugular venous waveform using a model of CSF dynamics, AJNR Am J Neuroradiol, 28 (2007) 983-989. [43] N.S. Elliott, D.A. Lockerby, A.R. Brodbelt, A lumped-parameter model of the cerebrospinal system for investigating arterial-driven flow in posttraumatic syringomyelia, Med Eng Phys, 33 (2011) 874-882.

[44] S. Marcotti, L. Marchetti, P. Cecconi, E. Votta, G.B. Fiore, A. Barberio, S. Viotti, A. Redaelli, M.M. Lagana, An anatomy-based lumped parameter model of cerebrospinal venous circulation: can an extracranial anatomical change impact intracranial hemodynamics?, BMC Neurol, 15 (2015) 95.

[45] T. Kim, S.G. Kim, Temporal dynamics and spatial specificity of arterial and venous blood volume changes during visual stimulation: implication for BOLD quantification, J Cereb Blood Flow Metab, 31 (2011) 1211-1222.

[46] N. Alperin, E.M. Vikingstad, B. Gomez-Anson, D.N. Levin, Hemodynamically independent analysis of cerebrospinal fluid and brain motion observed with dynamic phase contrast MRI, Magn Reson Med, 35 (1996) 741-754.

[47] A. Marmarou, K. Shulman, J. LaMorgese, Compartmental analysis of compliance and outflow resistance of the cerebrospinal fluid system, J Neurosurg, 43 (1975) 523-534.

[48] N. Alperin, M. Mazda, T. Lichtor, S.H. Lee, From cerebrospinal fluid pulsation to noninvasive intracranial compliance and pressure measured by MRI fow studies, Current Medical Imaging Reviews, 2 (2006) 117-129.

[49] N. Alperin, S.H. Lee, A.M. Bagci, MRI measurements of intracranial pressure in the upright posture: The effect of the hydrostatic pressure gradient, J Magn Reson Imaging, 42 (2015) 1158-1163.

[50] O. Baledent, L. Fin, L. Khuoy, K. Ambarki, A.C. Gauvin, C. Gondry-Jouet, M.E. Meyer, Brain hydrodynamics study by phase-contrast magnetic resonance imaging and transcranial color doppler, J Magn Reson Imaging, 24 (2006) 995-1004.

[51] A. Wahlin, K. Ambarki, R. Birgander, J. Malm, A. Eklund, Intracranial pulsatility is associated with regional brain volume in elderly individuals, Neurobiol Aging, 35 (2014) 365372.

[52] A. Wahlin, K. Ambarki, R. Birgander, N. Alperin, J. Malm, A. Eklund, Assessment of craniospinal pressure-volume indices, AJNR Am J Neuroradiol, 31 (2010) 1645-1650.

[53] K. Miller, K. Chinzei, Constitutive modelling of brain tissue: experiment and theory, Journal of Biomechanics, 30 (1997) 1115-1121.

[54] Y. Nakagawa, M. Tsuru, K. Yada, Site and mechanism for compression of the venous system during experimental intracranial hypertension, J Neurosurg, 41 (1974) 427-434.

[55] D. Greitz, Cerebrospinal fluid circulation and associated intracranial dynamics. A radiologic investigation using MR imaging and radionuclide cisternography, Acta Radiol Suppl, 386 (1993) 1-23.

[56] M. Kitano, W.H. Oldendorf, B. Cassen, The Elasticity of the Cranial Blood Pool, J Nucl Med, 5 (1964) 613-625.

[57] S. Gupta, M. Soellinger, D.M. Grzybowski, P. Boesiger, J. Biddiscombe, D. Poulikakos, V. Kurtcuoglu, Cerebrospinal fluid dynamics in the human cranial subarachnoid space: an 
overlooked mediator of cerebral disease. I. Computational model, J R Soc Interface, 7 (2010) 1195-1204.

[58] G.A. Bateman, The role of altered impedance in the pathophysiology of normal pressure hydrocephalus, Alzheimer's disease and syringomyelia, Med Hypotheses, 63 (2004) 980985.

[59] C.B. Beggs, Venous hemodynamics in neurological disorders: an analytical review with hydrodynamic analysis, BMC Med, 11 (2013) 142.

[60] C.B. Beggs, Cerebral venous outflow and cerebrospinal fluid dynamics, Veins and Lymphatics, 3 (2014) 1867.

[61] G.A. Bateman, The pathophysiology of idiopathic normal pressure hydrocephalus: cerebral ischemia or altered venous hemodynamics?, AJNR Am J Neuroradiol, 29 (2008) 198-203. 


\section{Tables}

Table 1. Mean, minimum and maximum flow rates (with the computed pulsatility and resistance indices) for the cervical arterial, venous and CSF flows, together with aqueductal CSF flow, aggregated for all subjects.

\begin{tabular}{lcccc} 
Signal & $\begin{array}{c}\text { Average } \\
(\mathbf{m L} / \mathbf{s}) \\
\text { Mean (SD) }\end{array}$ & $\begin{array}{c}\text { Caudal peak } \\
(\mathbf{m L} / \mathbf{s}) \\
\text { Mean (SD) }\end{array}$ & $\begin{array}{c}\text { Cranial peak } \\
(\mathbf{m L} / \mathbf{s}) \\
\text { Mean (SD) }\end{array}$ & $\begin{array}{c}\text { Pulsatility / } \\
\text { resistance index } \\
\text { Mean (SD) }\end{array}$ \\
\hline Arterial flow rate & $12.67(2.79)$ & $8.55(1.51)$ & $20.05(4.81)$ & $0.90(0.22)$ \\
Venous flow rate & $-12.67(1.53)$ & $-9.62(2.31)$ & $-15.62(2.24)$ & $0.49(0.21)$ \\
cCSF flow rate & $0.08(1.33)$ & $-2.83(1.08)$ & $1.83(0.61)$ & $8.31(133.55)$ \\
aCSF flow rate & $0.00(0.06)$ & $-0.10(0.06)$ & $0.10(0.05)$ & $10.69(63.91)$
\end{tabular}

cCSF - cervical cerebrospinal fluid (CSF) flow rate; aCSF - aqueductal CSF flow rate (i.e. CSF in the aqueduct of Sylvius).

Table 2. Pearson correlations between the average cumulative volume change signals in the cranium for the different fluids.

\begin{tabular}{lcccc} 
& Arterial & Venous & CSF & aCSF \\
\hline Arterial & 1 & & & \\
Venous & -0.818 & 1 & & \\
CSF & -0.997 & 0.820 & 1 & \\
aCSF & -0.664 & 0.967 & 0.676 & 1
\end{tabular}

$p$-value $<0.001$ for all the correlations

Table 3. Absolute timings expressed as fraction of the cardiac cycle, for minimum and maximum values of intracranial arterial, venous, CSF, and aqueductal CSF cumulative volume change signals.

\begin{tabular}{lccc}
$\begin{array}{c}\text { Intracranial cumulative } \\
\text { volume change } \\
\text { (max/min) }\end{array}$ & $\begin{array}{c}\text { Absolute timing } \\
\text { (fraction of CC) } \\
\text { Mean (SD) }\end{array}$ & $\begin{array}{c}\text { Delay from } \\
\text { minimum arterial } \\
\text { cumulative } \\
\text { volume change } \\
\text { Mean (SD) }\end{array}$ & $\begin{array}{c}\text { Delay from } \\
\text { maximum venous } \\
\text { cumulative } \\
\text { volume change } \\
\text { Mean (SD) }\end{array}$ \\
\hline Arterial (maximum) & $0.974(0.098)$ & $0.417(0.052)$ & $0.359(0.067)$ \\
Arterial (minimum) & $0.557(0.084)$ & $0.000(0.000)$ & $0.943(0.059)$ \\
Venous (maximum) & $0.615(0.062)$ & $0.057(0.059)$ & $0.000(0.000)$ \\
Venous (minimum) & $0.148(0.122)$ & $0.591(0.099)$ & $0.534(0.076)$ \\
CSF (maximum) & $0.573(0.080)$ & $0.016(0.056) \#$ & $0.958(0.068) \#$ \\
CSF (minimum) & $0.995(0.084)$ & $0.438(0.058)$ & $0.380(0.076)$ \\
Aqueductal CSF (maximum) & $0.685(0.109)$ & $0.128(0.083)$ & $0.070(0.092)$ \\
Aqueductal CSF (minimum) & $0.154(0.104)$ & $0.596(0.065)$ & $0.539(0.081)$
\end{tabular}

CSF - cerebrospinal fluid

All delays are significantly different with the exception of \#(p=0.352) and \#\# $(p=0.063)$ 


\section{Figures}
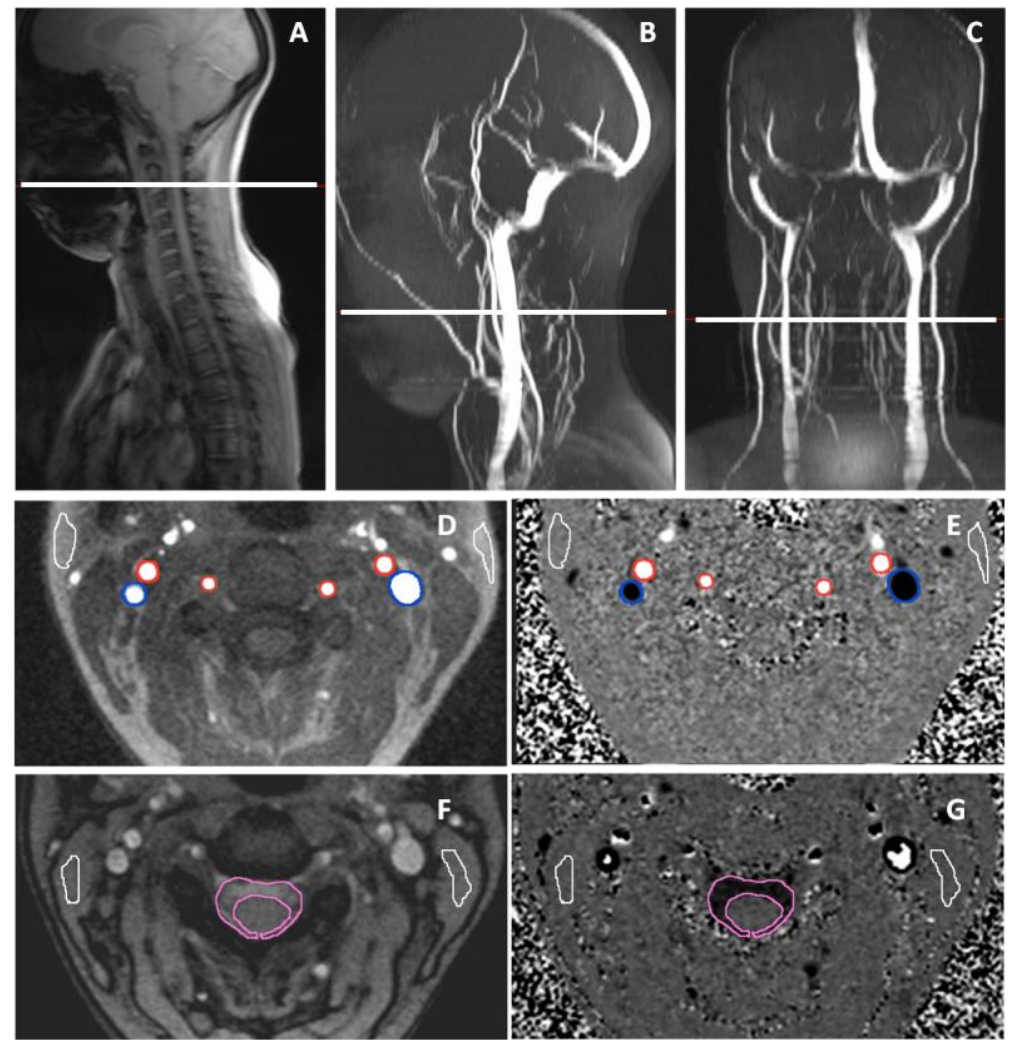

Figure 1. Phase contrast (PC) images at C2/C3 cervical level. Sagittal T1 neck localizer (a), TOF Maximum Intensity Projection on the sagittal (b) and coronal (c) planes were used for the positioning of PC slices. The Internal Jugular Veins, Vertebral Veins, Internal Carotid Arteries, Vertebral Arteries were segmented on the magnitude image of the high-venc PC (d) and the same region of interests (ROls) were superimposed to the phase image (e). For the cCSF measure, the subarachnoid space at cervical level was segmented on the phase image of the low-venc PC (g), using the magnitude image (f) to increase the confidence of the contours. The ROls drawn in stationary structures for the estimation of the phase offset are also shown.

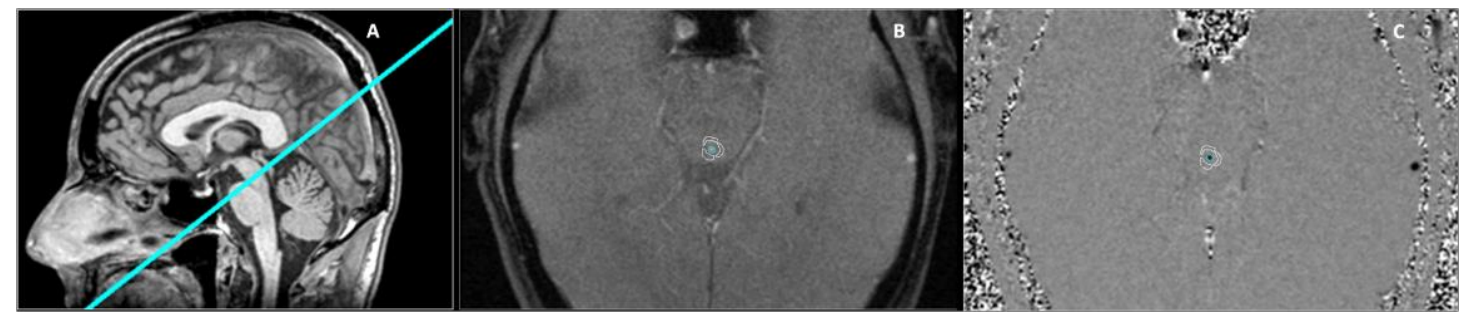

Figure 2. Phase contrast images at aqueductal level. Sagittal T1 brain localizer (a), was used for the positioning of PC slice. The ROI drawn on the PC magnitude (b) and phase (c) for the aCSF measure and the ROls drawn in stationary structures for the estimation of the phase offset are shown. 


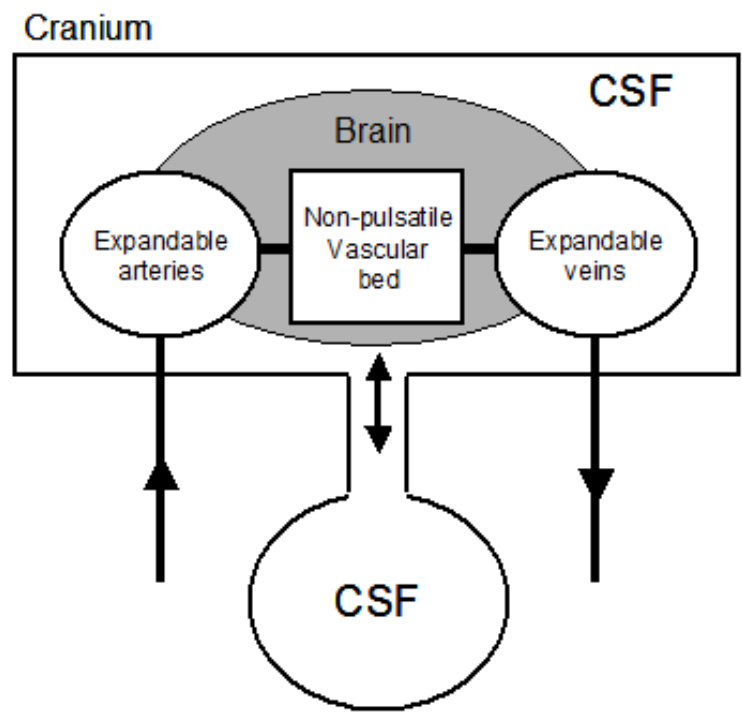

Figure 3. Schematic diagram of the intracranial vascular model.

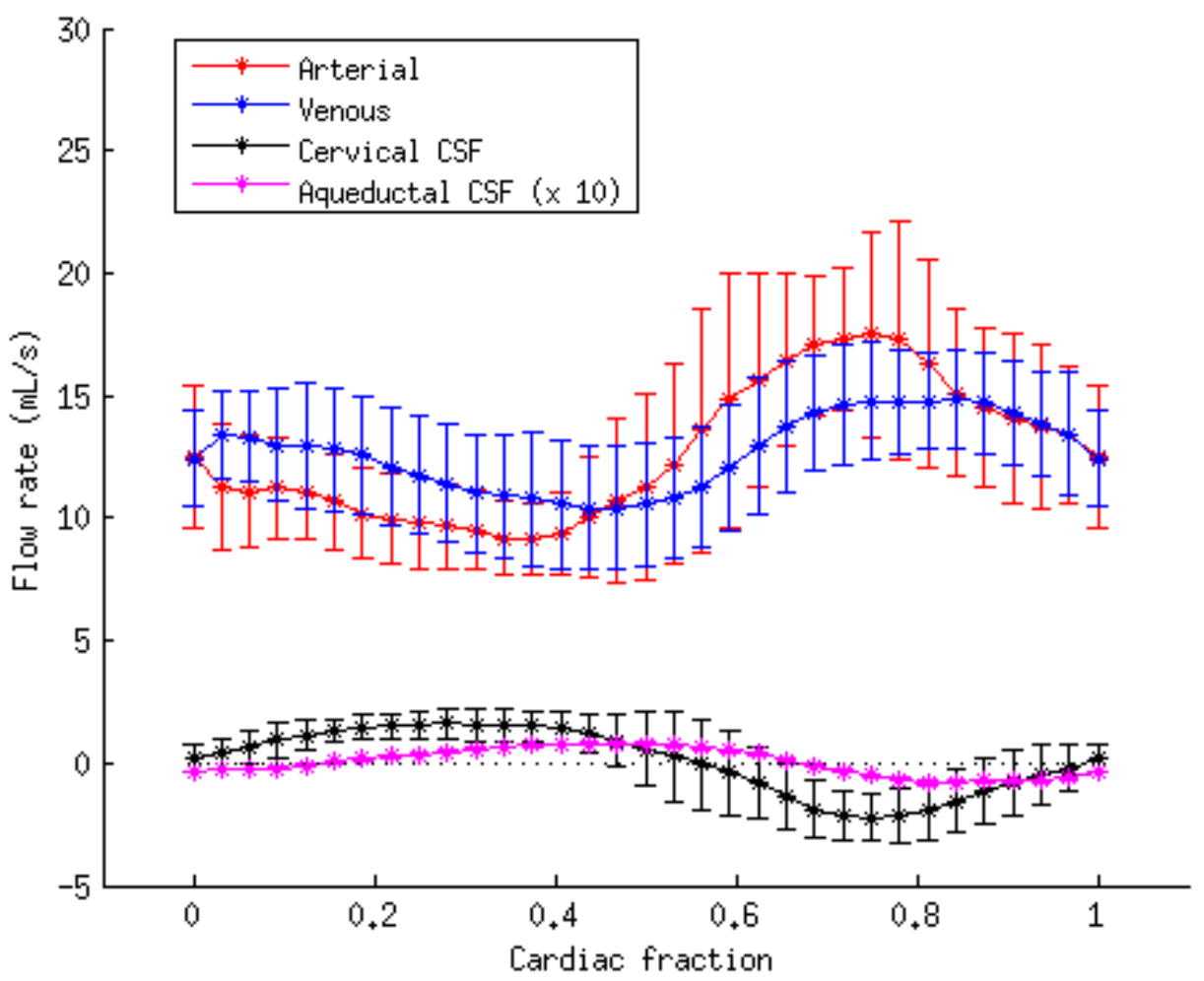

Figure 4. Cervical mean arterial, venous, cCSF and aCSF flow rates over a cardiac cycle, for all twelve subjects aggregated together. Error bars represent one standard deviation. (NB. For ease of representation the venous signal has been inverted.) 


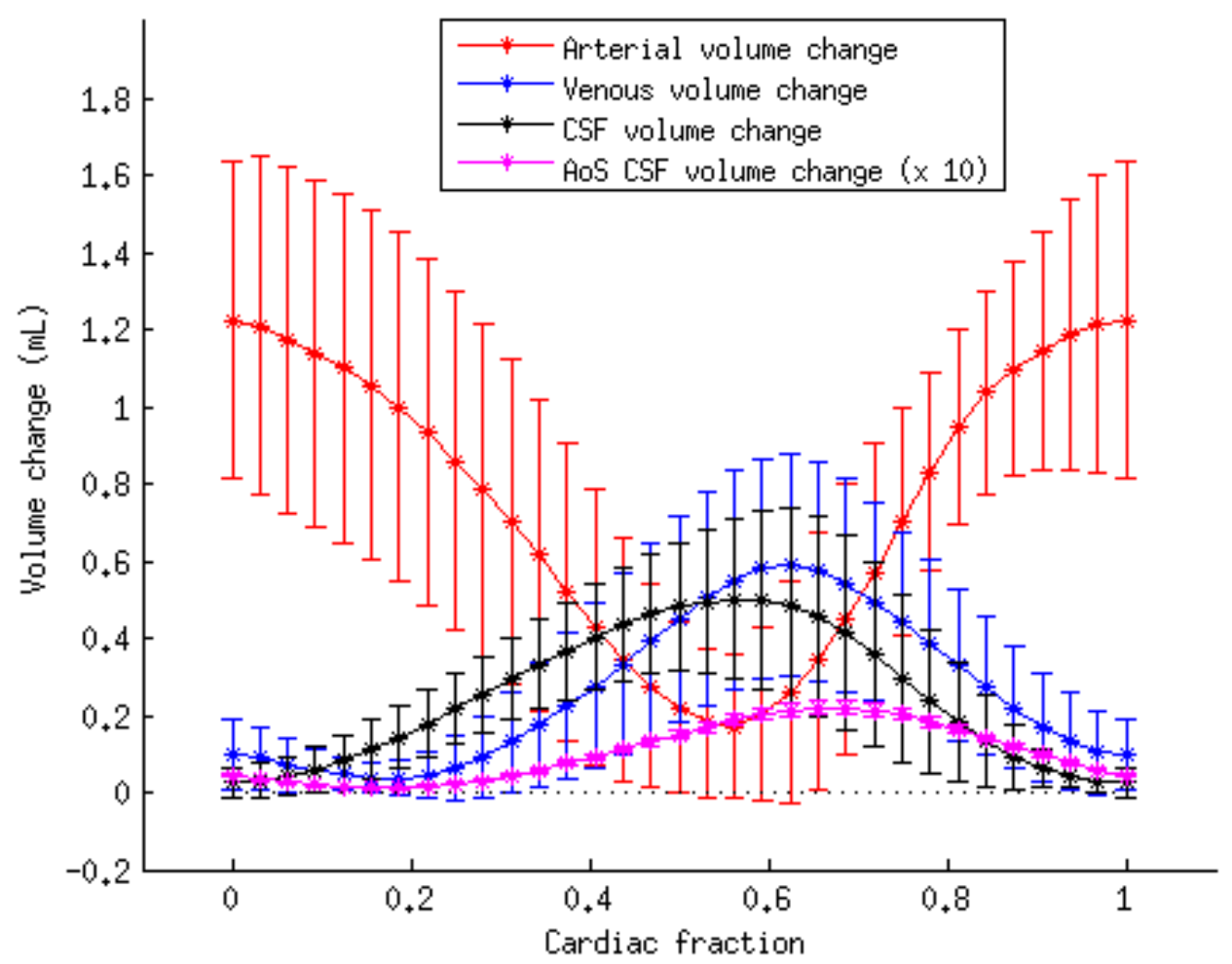

Figure 5. Mean intracranial arterial, venous, CSF and aCSF volume changes over the cardiac cycle, for all twelve subjects aggregated together. Error bars represent one standard deviation.

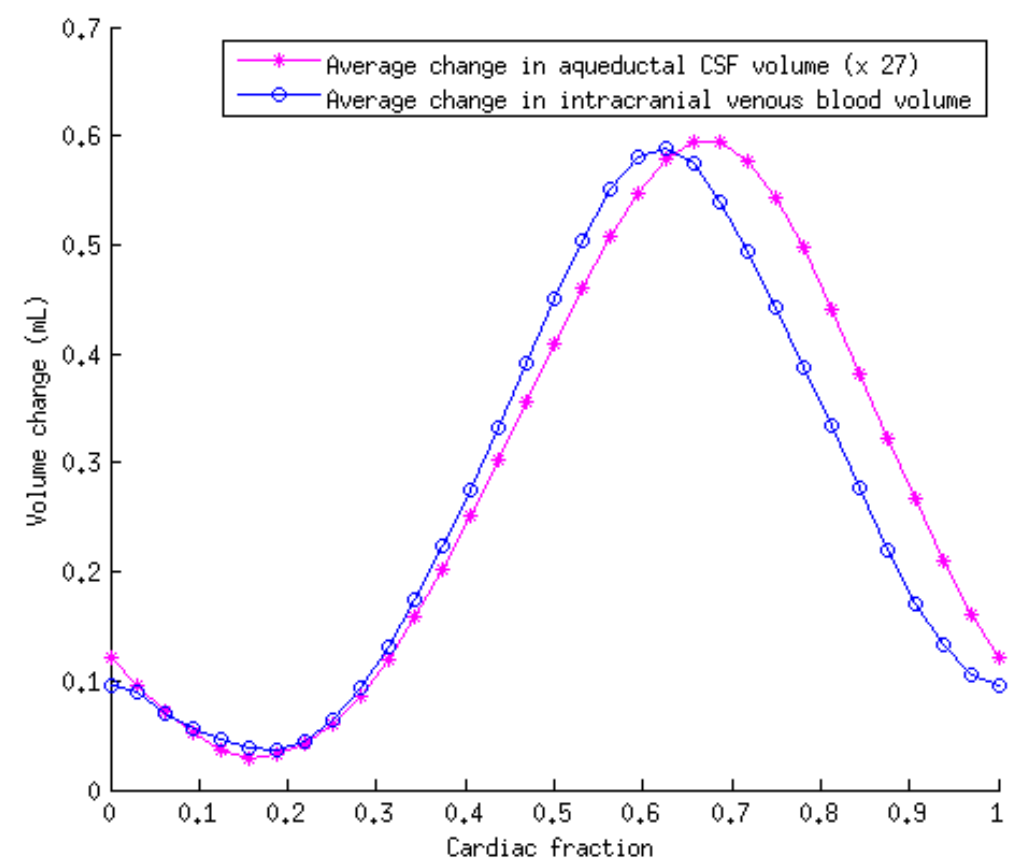

Figure 6. Mean intracranial venous and aCSF volume changes over the cardiac cycle, scaled so that $\mathrm{t}$
$\mathrm{h}$
$\mathrm{e}$ 